\title{
Boundedness of solutions for semilinear Duffing's equation with asymmetric nonlinear term
}

Shunjun Jiang ${ }^{1 *}$, Feng Rao ${ }^{1}$ and Yanling Shi ${ }^{2}$

\section{"Correspondence:}

jiangshunjun@njut.edu.cn

${ }^{1}$ College of Sciences, Nanjing

University of Technology, Nanjing,

210009, People's Republic of China

Full list of author information is

available at the end of the article

\begin{abstract}
In this paper we study the following second-order periodic system:

$$
x^{\prime \prime}+V^{\prime}(x)+p(x) f(t)=0,
$$

where $V(x)$ has a singularity. Under some assumptions on the $V(x), p(x)$ and $f(t)$, by Ortega's small twist theorem, we obtain the existence of quasi-periodic solutions and boundedness of all the solutions.
\end{abstract}

Keywords: boundedness of solutions; singularity; small twist theorem

\section{Introduction and main result}

In the early 1960s, Littlewood [1] asked whether or not the solutions of the Duffing-type equations,

$$
x^{\prime \prime}+g(x, t)=0
$$

are bounded for all time, i.e., whether there are resonances that might cause the amplitude of the oscillations to increase without bound.

The first positive result of boundedness of solutions in the superlinear case $\left(i . e ., \frac{g(x, t)}{x} \rightarrow\right.$ $\infty$ as $|x| \rightarrow \infty)$ was due to Morris [2]. By means of KAM theorem, Morris proved that every solution of differential equation (1.1) is bounded if $g(x, t)=2 x^{3}-p(t)$, where $p(t)$ is piecewise continuous and periodic. This result relies on the fact that the nonlinearity $2 x^{3}$ can guarantee the twist condition of KAM theorem. Later, several authors (see $[3,4]$ ) improved the result of (1.1) and obtained a similar result for a large class of superlinear functions $g(x, t)$.

When $g(x, t)$ satisfies

$$
0 \leq k \leq \frac{g(x, t)}{x} \leq K \leq+\infty, \quad \forall x \in R
$$

i.e., differential equation (1.1) is semilinear, similar results also hold. But the proof is more difficult since there may be a resonant case.

\section{Springer}

O2013 Jiang et al.; licensee Springer. This is an Open Access article distributed under the terms of the Creative Commons Attribution License (http://creativecommons.org/licenses/by/2.0), which permits unrestricted use, distribution, and reproduction in any medium, provided the original work is properly cited. 
Liu [5] studied the following equation:

$$
\left(\Phi_{p}\left(x^{\prime}\right)\right)^{\prime}+\alpha \Phi_{p}\left(x^{+}\right)+\beta \Phi_{p}\left(x^{-}\right)=f(x, t)
$$

where $f(x, t)$ is $2 \pi$-periodic in $t$ and has limits $f_{ \pm}(t)$ as $x \rightarrow \pm \infty$. Under some reasonable assumptions on $f(x, t)$, Liu [5] proved the existence of quasi-periodic solutions and the boundedness of solutions. Later, Cheng and $\mathrm{Xu}[6]$ studied a more general equation

$$
\left(\Phi_{p}\left(x^{\prime}\right)+\Psi\left(x^{\prime}\right)\right)^{\prime}+\alpha \Phi_{p}\left(x^{+}\right)+\beta \Phi_{p}\left(x^{-}\right)=\phi(x, t)
$$

where $\phi(x, t)$ is $2 \pi$-periodic in $t$. They defined a new function $\bar{\phi}(t, x)=\frac{\phi(t, x)}{|x|^{p-1-\sigma}}$, where $\sigma \in$ $(0, p), \bar{\phi}(t, x)$ has limits $\phi_{ \pm}(t)$ and the similar property to $f(x, t)$ in [5]. Then the authors proved the boundedness of solutions for (1.2). We observe that $\phi(x, t)$ in [6] is unbounded while $f(x, t)$ in [5] is bounded and that is the major difference between [5] and [6]. The idea in $[5,6]$ is to change the original problem to a Hamiltonian system and then use a twist theorem of area-preserving mapping to the Poincaré map.

Recently, Capietto et al. [7] studied the following equation:

$$
x^{\prime \prime}+V^{\prime}(x)=F(x, t)
$$

where $F(x, t)=p(t)$ is a $\pi$-periodic function, $V(x)=\frac{1}{2} x_{+}^{2}+\frac{1}{\left(1-x_{-}^{2}\right)^{v}}-1, x_{+}=\max \{x, 0\}, x_{-}=$ $\max \{-x, 0\}$ and $v$ is a positive integer. Under the Lazer-Leach assumption that

$$
1+\frac{1}{2} \int_{0}^{\pi} p\left(t_{0}+\theta\right) \sin \theta d \theta>0, \quad \forall t_{0} \in R
$$

they proved the boundedness of solutions and the existence of a quasi-periodic solution by the Moser twist theorem. It was the first time that the equation of the boundedness of all solutions was treated in case of a singular potential.

Motivated by the papers [5-7], we observe that $F(x, t)=p(t)$ in (1.3) is smooth and bounded, so a natural question is to find sufficient conditions on $F(x, t)$ such that all solutions of (1.3) are bounded when $F(x, t)$ is unbounded. The purpose of this paper is to deal with this problem.

We consider the following equation:

$$
x^{\prime \prime}+V^{\prime}(x)+p(x) f(t)=0
$$

where

$$
V=\frac{1}{2} x_{+}^{2}+\frac{1}{1-x_{-}^{2}}-1, \quad x>-1
$$

In order to state our main results, we give some notations and assumptions. Let $f(t)$ be a $\pi$-periodic function and

$$
\lim _{x \rightarrow+\infty} \frac{p(x)}{|x|^{\alpha}}=1, \quad P(x)=\int_{0}^{x} p(s) d s,
$$


where $0<\alpha<1$. We suppose that the following Lazer-Leach assumption holds:

$$
\int_{0}^{\pi} f\left(t_{0}+\theta\right)(\sin \theta)^{1+\alpha} d \theta>0, \quad \forall t_{0} \in R
$$

Our main result is the following theorem.

Theorem 1 Under assumptions (1.6)-(1.8), all the solutions of (1.5) are defined for all $t \in$ $(-\infty,+\infty)$, and for each solution $x(t)$, we have $\sup _{t \in R}\left(|x(t)|+\left|x^{\prime}(t)\right|\right)<+\infty$.

The main idea of our proof is acquired from [8]. The proof of Theorem 1 is based on a small twist theorem due to Ortega [9]. Hypotheses (1.6)-(1.8) of our theorem are used to prove that the Poincaré mapping of (1.5) satisfies the assumptions of Ortega's theorem.

Moreover, we have the following theorem on solutions of Mather type.

Theorem 2 Assume that $f(t) \in C$ satisfies (1.8); then, there is $\epsilon_{0}>0$ such that for any $\omega \in$ $\left(\frac{1}{\pi}, \frac{1}{\pi+\epsilon_{0}}\right)$, equation (1.5) has a solution $\left(x_{\omega}(t), x_{\omega}^{\prime}(t)\right)$ of Mather type with rotation number $\omega$. More precisely,

Case 1: $\omega=\frac{p}{q}$ is rational. The solutions $\left(x_{\omega}(t+2 i \pi), x_{\omega}^{\prime}(t+2 i \pi)\right), 1 \leq i \leq q-1$, are independent periodic solutions of periodic $q \pi ;$ moreover, in this case,

$$
\lim _{q \rightarrow \infty} \min _{t \in R}\left(\left|x_{\omega}(t)\right|+\left|x_{\omega}^{\prime}(t)\right|\right)=+\infty
$$

Case 2: $\omega$ is irrational. The solution $\left(x_{\omega}(t), x_{\omega}^{\prime}(t)\right)$ is either a usual quasi-periodic solution or a generalized one.

\section{Proof of the theorem}

\subsection{Action-angle variables and some estimates}

Observe that (1.5) is equivalent to the following Hamiltonian system:

$$
x^{\prime}=\frac{\partial H}{\partial y}, \quad y^{\prime}=-\frac{\partial H}{\partial x}
$$

with the Hamiltonian function

$$
H(x, y, t)=\frac{1}{2} y^{2}+V(x)+P(x) f(t) .
$$

In order to introduce action and angle variables, we first consider the auxiliary autonomous equation

$$
x^{\prime}=y, \quad y^{\prime}=-V^{\prime}(x),
$$

which is an integrable Hamiltonian system with the Hamiltonian function

$$
H_{1}(x, y, t)=\frac{1}{2} y^{2}+V(x) .
$$

The closed curves $H_{1}(x, y, t)=h>0$ are just the integral curves of (2.2). 
Denote by $T_{0}(h)$ the time period of the integral curve $\Gamma_{h}$ of $(2.2)$ defined by $H_{1}(x, y, t)=h$ and by $I$ the area enclosed by the closed curve $\Gamma_{h}$ for every $h>0$. Let $-1<-\alpha_{h}<0<\beta_{h}$ be such that $V\left(-\alpha_{h}\right)=V\left(\beta_{h}\right)=h$. It is easy to see that

$$
I_{0}(h)=2 \int_{-\alpha_{h}}^{\beta_{h}} \sqrt{2(h-V(s))} d s, \quad \forall h>0,
$$

and

$$
T_{0}(h)=I_{0}^{\prime}(h)=2 \int_{\alpha_{h}}^{\beta_{h}} \frac{1}{\sqrt{2(h-V(s))}} d s, \quad \forall h>0 .
$$

By a direct computation, we get

$$
\begin{aligned}
I_{0}(h) & =2 \int_{0}^{\beta_{h}} \sqrt{2(h-V(s))} d s+2 \int_{-\alpha_{h}}^{0} \sqrt{2(h-V(s))} d s \\
& =\pi h+2 \int_{0}^{\alpha_{h}} \sqrt{2(h-V(-s))} d s,
\end{aligned}
$$

so

$$
T_{0}(h)=\pi+\int_{0}^{\alpha_{h}} \frac{1}{\sqrt{2(h-V(-s))}} d s .
$$

We then have

$$
I_{0}(h)=I_{-}(h)+I_{+}(h), \quad T_{0}(h)=T_{-}(h)+T_{+}(h),
$$

where

$$
\begin{array}{ll}
I_{-}(h)=2 \int_{0}^{-\alpha_{h}} \sqrt{2(h-V(s))} d s, & I_{+}(h)=\pi h, \\
T_{-}(h)=2 \int_{0}^{-\alpha_{h}} \frac{1}{\sqrt{2(h-V(-s))}} d s, & T_{+}(h)=\pi .
\end{array}
$$

We now give the estimates on the functions $I_{-}$and $T_{-}$.

Lemma 1 We have

$$
h^{n}\left|\frac{d^{n} T_{-}(h)}{d h^{n}}\right| \leq C h^{-\frac{1}{2}},
$$

and

$$
h^{n}\left|\frac{d^{n} I_{-}(h)}{d h^{n}}\right| \leq C h^{\frac{1}{2}}
$$

where $n=0,1, \ldots, 6, h \rightarrow+\infty$. Note that here and below we always use $C, C_{0}$ or $C_{0}^{\prime}$ to indicate some constants. 
Proof Now we estimate the first inequality. We choose $\frac{V(s)}{h}=\eta$ as the new variable of integration, then we have

$$
T_{-}(h)=\int_{-\alpha_{h}}^{0} \frac{1}{\sqrt{2(h-V(s))}} d s=\int_{0}^{1} \frac{\sqrt{h}}{V^{\prime}(s(\eta, h))} \frac{1}{\sqrt{2(1-\eta)}} d \eta .
$$

Since $V(s)=\frac{1}{1-s^{2}}-1$ and $\frac{V(s)}{h}=\eta$, we have $s=\sqrt{\frac{\eta h}{1+\eta h}}$. By a direct computation, we have

$$
V^{\prime}(s)=\frac{2 s}{\left(1-s^{2}\right)^{2}}=\frac{2 \sqrt{\eta h}(1+\eta h)^{2}}{\sqrt{1+\eta h}}
$$

then we get

$$
T_{-}^{(n)}(h)=\frac{\left(-\frac{3}{2}\right) !}{\left(-\frac{3}{2}-n\right) !} \int_{0}^{1} \frac{\eta^{n}}{\sqrt{2 \eta(1-\eta)}(1+\eta h)^{\frac{3}{2}+n}} d \eta, \quad n=0,1, \ldots, 6 .
$$

When $0 \leq \eta \leq h^{-1}$ and $h$ is sufficient large, there exits $C_{0}$ such that $1-\eta>C_{0}$, so we have

$$
\begin{aligned}
\int_{0}^{h^{-1}} \frac{\eta^{n}}{\sqrt{2 \eta(1-\eta)}(1+\eta h)^{\frac{3}{2}+n}} d \eta & \leq C \int_{0}^{h^{-1}} \frac{\eta^{n}}{\sqrt{2 \eta(1-\eta)}} d \eta \\
& \leq \frac{C}{C_{0}} \int_{0}^{h^{-1}} \eta^{n-\frac{1}{2}} d \eta \leq C h^{-\frac{1}{2}-n}
\end{aligned}
$$

Since $h^{-\frac{2}{3}} \leq \eta \leq 1$, we have

$$
h^{\frac{1}{3}}<1+h^{\frac{1}{3}} \leq 1+\eta h \leq 1+h
$$

then

$$
\begin{aligned}
\int_{h^{-\frac{2}{3}}}^{1} \frac{\eta^{n}}{\sqrt{2 \eta(1-\eta)}(1+\eta h)^{\frac{3}{2}+n}} d \eta & \leq C \int_{h^{-\frac{2}{3}}}^{1} \frac{\eta^{n} h^{n}}{\sqrt{2 \eta(1-\eta)} h^{n}(1+\eta h)^{n}(1+\eta h)^{\frac{3}{2}}} d \eta \\
& \leq C \int_{h^{-\frac{2}{3}}}^{1} \frac{1}{\sqrt{2 \eta(1-\eta)} h^{n}(1+\eta h)^{\frac{3}{2}}} d \eta \\
& \leq C \int_{h^{-\frac{2}{3}}}^{1} \frac{1}{\sqrt{2 \eta(1-\eta)} h^{n} h^{\frac{1}{2}}} d \eta \\
& \leq C h^{-\frac{1}{2}-n} \int_{0}^{1} \frac{1}{\sqrt{2 \eta(1-\eta)}} d \eta \leq C h^{-\frac{1}{2}-n} .
\end{aligned}
$$

Observing that there is $C_{0}>0$ such that $\sqrt{1-\eta} \geq C_{0}$ when $h^{-1} \leq \eta \leq h^{-\frac{2}{3}}$ and $h \rightarrow+\infty$, we have

$$
\begin{aligned}
& \int_{h^{-1}}^{h^{-\frac{2}{3}}} \frac{\eta^{n}}{\sqrt{2 \eta(1-\eta)}(1+\eta h)^{\frac{3}{2}+n}} d \eta \\
& \quad \leq C_{1} h^{-\frac{3}{2}-n} \int_{h^{-1}}^{h^{-\frac{2}{3}}} \frac{1}{\sqrt{2 \eta(1-\eta)} \eta^{\frac{3}{2}}} d \eta
\end{aligned}
$$




$$
\begin{aligned}
& \leq \frac{C_{1}}{C_{0}} h^{-\frac{3}{2}-n} \int_{h^{-1}}^{h^{-\frac{2}{3}}} \frac{1}{\eta^{2}} d \eta=\left.\frac{C_{1}}{C_{0}} h^{-\frac{3}{2}-n} \frac{1}{\eta}\right|_{h_{-1}} ^{h^{-\frac{2}{3}}} \\
& =\frac{C_{1}}{C_{0}} h^{-\frac{3}{2}-n}\left(h-h^{\frac{2}{3}}\right) \leq C h^{-\frac{1}{2}-n} .
\end{aligned}
$$

By (2.3)-(2.5) we have $T_{-}^{(n)}(h) \leq C h^{-\frac{1}{2}-n}, n=0,1, \ldots, 6$.

The proof of the second inequality is similar to that of the first one, so we only give a brief proof. We choose $\frac{V(s)}{h}=\eta$ as the new variable of integration, so we have

$$
\frac{\partial s}{\partial h}=\frac{\eta}{V^{\prime}}, \quad s=\sqrt{\frac{\eta h}{1+\eta h}}
$$

and

$$
V^{\prime}(s)=\frac{2 s}{\left(1-s^{2}\right)^{2}}=\frac{2 \sqrt{\eta h}(1+\eta h)^{2}}{\sqrt{1+\eta h}} .
$$

By a direct computation, we have

$$
I_{-}(h)=2 \int_{-\alpha_{h}}^{0} \sqrt{2(h-V(s))} d s=h \int_{0}^{1} \frac{\sqrt{2(1-\eta)}}{\sqrt{\eta}(1+\eta h)^{\frac{3}{2}}} d \eta .
$$

By (2.6), we can easily get

$$
\begin{aligned}
I_{-}^{(n)}(h)= & I_{-1}^{(n)}(h)+I_{-2}^{(n)}(h)=n \frac{\left(-\frac{3}{2}\right) !}{\left(-\frac{3}{2}-n+1\right) !} \int_{0}^{1} \frac{\sqrt{2(1-\eta)}}{\sqrt{\eta}} \frac{\eta^{n-1}}{(1+\eta h)^{\frac{3}{2}+n-1}} d \eta \\
& +\frac{\left(-\frac{3}{2}\right) !}{\left(-\frac{3}{2}-n\right) !} h \int_{0}^{1} \frac{\sqrt{2(1-\eta)}}{\sqrt{\eta}} \frac{\eta^{n}}{(1+\eta h)^{\frac{3}{2}+n}} d \eta
\end{aligned}
$$

where $n=0,1, \ldots, 6$.

By a similar way to that in estimating $T_{-}^{(n)}(h)$, we get

$$
I_{-1}^{(n)}(h) \leq C h^{\frac{1}{2}-n}, \quad I_{-2}^{(n)}(h) \leq C h^{\frac{1}{2}-n},
$$

which means that

$$
I_{-}^{(n)}(h) \leq C h^{\frac{1}{2}-n}, \quad n=0,1, \ldots, 6 .
$$

Thus Lemma 1 is proved.

Remark 1 It follows from the definitions of $T_{+}(h), T_{-}(h)$ and Lemma 1 that

$$
\lim _{h \rightarrow+\infty} T_{-}(h)=0, \quad \lim _{h \rightarrow+\infty} T_{+}(h)=\pi .
$$

Thus the time period $T_{0}(h)$ is dominated by $T_{+}(h)$ when $h$ is sufficiently large. From the relation between $T_{-}(h)$ and $I_{-}(h)$, we know $I_{0}(h)$ is dominated by $I_{+}(h)$ when $h$ is sufficiently large. 
Remark 2 It also follows from the definition of $I(h), I_{-}(h), I_{+}(h)$ and Remark 1 that

$$
\left|h^{n} \frac{d^{n} I_{0}(h)}{d h^{n}}\right| \leq C_{0} I_{0}(h) \quad \text { for } n \geq 1
$$

Remark 3 Note that $h=h_{0}\left(I_{0}\right)$ is the inverse function of $I_{0}$. By Remark 2, we have

$$
\left|I^{n} \frac{d^{n} h(I)}{d I^{n}}\right| \leq C_{0} h(I) \quad \text { for } n \geq 1
$$

We now carry out the standard reduction to the action-angle variables. For this purpose, we define the generating function $S(x, I)=\int_{C} \sqrt{2(h-V(s))} d s$, where $C$ is the part of the closed curve $\Gamma_{h}$ connecting the point on the $y$-axis and point $(x, y)$.

We define the well-know map $(\theta, I) \rightarrow(x, y)$ by

$$
y=\frac{\partial S}{\partial x}(x, I), \quad \theta=\frac{\partial S}{\partial I}(x, I),
$$

which is symplectic since

$$
\begin{aligned}
& d x \wedge d y=d x \wedge\left(S_{x x} d x+S_{x I} d I\right)=S_{x I} d x \wedge d I \\
& d \theta \wedge d I=\left(S_{I x} d x+S_{I I} d I\right) \wedge d I=S_{I x} d x \wedge d I
\end{aligned}
$$

From the above discussion, we can easily get

$$
\theta= \begin{cases}\frac{\pi}{T_{0}(h(x, y))}\left(\frac{T_{-}(h(x, y))}{2}+\arcsin \frac{x}{\sqrt{2(h(x, y))}}\right) & \text { if } x>0, y>0 \\ \frac{\pi}{T_{0}(h(x, y))}\left(\frac{T_{-}(h(x, y))}{2}+\pi+\arcsin \frac{x}{\sqrt{2(h(x, y))}}\right) & \text { if } x>0, y<0 \\ \frac{\pi}{T_{0}(h(x, y))}\left(\int_{-\alpha_{h}}^{x} \frac{1}{\sqrt{2\left(h(x, y)+1-\left(1-s^{2}\right)^{-1}\right)}} d s\right) & \text { if } x<0, y>0 \\ \frac{\pi}{T_{0}(h(x, y))}\left(T_{0}(h(x, y))-\int_{-\alpha_{h}}^{x} \frac{1}{\sqrt{2\left(h(x, y)+1-\left(1-s^{2}\right)^{-1}\right)}} d s\right) & \text { if } x<0, y<0\end{cases}
$$

and

$$
I(x, y)=I_{0}(h(x, y))=2 \int_{-\alpha_{h}}^{\beta_{h}} \sqrt{2(h(x, y)-V(s))} d s .
$$

In the new variables $(\theta, I)$, system (2.1) becomes

$$
\theta^{\prime}=\frac{\partial H}{\partial I}, \quad I^{\prime}=-\frac{\partial H}{\partial \theta},
$$

where

$$
H(\theta, I, t)=\pi h_{0}(I)+\pi P(x(I, \theta)) f(t) .
$$

In order to estimate $\pi P(I, \theta)$, we need the estimate on the functions $x(I, \theta)$.

Lemma 2 For I sufficient large and $-\alpha_{h} \leq x<0$, the following estimates hold:

$$
\left|I^{n} \frac{\partial^{n} x(I, \theta)}{\partial I^{n}}\right| \leq c \sqrt{I} \quad \text { for } 0 \leq n \leq 6
$$


The lemma was first proved in [3], later Capietto et al. [7] gave a different proof; using the method of induction-hypothesis, Jiang and Fang [10] also gave another proof. So, for concision, we omit the proof.

\subsection{New action and angle variables}

Now we are concerned with Hamiltonian system (2.10) with the Hamiltonian function $H(\theta, I, t)$ given by $(2.11)$. Note that

$$
I d \theta-H d t=-(H d t-I d \theta) \text {. }
$$

This means that if one can solve $I$ from (2.11) as a function of $H$ ( $\theta$ and $t$ as parameters), then

$$
\frac{d H}{d \theta}=-\frac{\partial I}{\partial t}(t, H, \theta), \quad \frac{d t}{d \theta}=\frac{\partial I}{\partial H}(t, H, \theta)
$$

is also a Hamiltonian system with the Hamiltonian function $I$ and now the action, angle and time variables are $H, t$ and $\theta$.

From (2.11) and Lemma 1, we have

$$
\frac{\partial H}{\partial I} \rightarrow 1 \quad \text { as } I \rightarrow+\infty
$$

So, we assume that $I$ can be written as

$$
I=I_{0}\left(\frac{H}{\pi}+R(H, t, \theta)\right)
$$

where $R$ satisfies $|R|<\frac{H}{\pi}$. Recalling that $h_{0}$ is the inverse function of $I_{0}$, we have

$$
\frac{H}{\pi}+R(H, t, \theta)=h_{0}(I)
$$

which implies that

$$
R(H, t, \theta)=P(x(I, \theta)) f(t) .
$$

As a consequence, $R$ is implicitly defined by

$$
R(H, t, \theta)=P\left[x\left(I_{0}\left(\frac{H}{\pi}+R(H, t, \theta)\right), \theta\right)\right] f(t) .
$$

Now we give the estimates of $R$. By a similar way to that in estimating Lemma 2.3 in [7], we have the following lemma.

Lemma 3 The function $R(H, t, \theta)$ satisfies the following estimates:

$$
\left|\frac{\partial^{m+l} R(H, t, \theta)}{\partial H^{m} \partial t^{l}}\right| \leq H^{\frac{\alpha+1}{2}} \quad \text { for } m+l \leq 6
$$


Moreover, by the implicit function theorem, there exists a function $R_{1}=R_{1}(t, H, \theta)$ such that

$$
R(H, t, \theta)=P(x(H, \theta)) f(t)+R_{1}(H, t, \theta) .
$$

Since

$$
\begin{aligned}
R_{1}(H, t, \theta)= & R(H, t, \theta)-P(x(H, \theta)) f(t) \\
= & P\left\{x\left[I_{0}\left(\frac{H}{\pi}+R(H, t, \theta)\right), \theta\right]\right\} f(t)-P(x(H, \theta)) f(t) \\
= & \int_{0}^{1} p\left\{x\left[H+s\left(\pi R+I_{-}\right), \theta\right]\right\} \\
& \cdot \frac{\partial x}{\partial I}\left(H+s\left(\pi R+I_{-}\right), \theta\right) \cdot\left(\pi R+I_{-}\right) f(t) d s .
\end{aligned}
$$

By Lemmas 1 and 3, we have the estimates on $R_{1}(H, t, \theta)$.

Lemma $4\left|\frac{\partial^{k+l} R_{1}(H, t, \theta)}{\partial^{k} H \partial^{l} t}\right|<H^{\frac{\alpha}{2}}$ for $k+l \leq 6$.

For the estimate of $I\left(\frac{H}{\pi}+R\right)$, we need the estimate on $I_{-}\left(\frac{H}{\pi}+R\right)$. By Lemma 1 and noticing that $|R|<\frac{H}{\pi}$, we have the following lemma.

Lemma $5\left|\frac{\partial^{k+l} I_{-}\left(\frac{H}{\pi}+R\right)}{\partial^{k} H \partial^{l} t}\right|<H^{\frac{1}{2}}$ for $k+l \leq 6$.

Now the new Hamiltonian function $I=I(t, H, \theta)$ is written in the form

$$
\begin{aligned}
I & =I_{0}\left(\frac{H}{\pi}+R\right)=I_{+}\left(\frac{H}{\pi}+R\right)+I_{-}\left(\frac{H}{\pi}+R\right) \\
& =H+\pi R(H, t, \theta)+I_{-}\left(\frac{H}{\pi}+R\right) \\
& =H+\pi P(x(H, \theta)) f(t)+\pi R_{1}(H, t, \theta)+I_{-}\left(\frac{H}{\pi}+R\right) .
\end{aligned}
$$

System (2.12) is of the form

$$
\left\{\begin{array}{l}
\frac{d t}{d \theta}=\frac{\partial I}{\partial H}=1+\pi \frac{\partial x}{\partial H}(H, \theta) p(x(H, \theta)) f(t)+\pi \frac{\partial R_{1}}{\partial H}(H, t, \theta)+\frac{\partial I_{-}}{\partial H}(H, t, \theta), \\
\frac{d H}{d \theta}=-\frac{\partial I}{\partial t}=-\pi P(x(\theta, H)) f^{\prime}(t)-\pi \frac{\partial R_{1}}{\partial t}(t, H, \theta)-\frac{\partial I_{-}}{\partial t}(H, t, \theta) .
\end{array}\right.
$$

Introduce a new action variable $\rho \in[1,2]$ and a parameter $\epsilon>0$ by $H=\epsilon^{-2} \rho$. Then $H \gg$ $1 \Leftrightarrow 0<\epsilon \ll 1$. Under this transformation, system (2.14) is changed into the form

$$
\left\{\begin{array}{l}
\frac{d t}{d \theta}=\frac{\partial I}{\partial H}=1+\pi \frac{\partial x}{\partial H}(H, \theta) p(x(H, \theta)) f(t)+\pi \frac{\partial R_{1}}{\partial H}(H, t, \theta)+\frac{\partial I_{-}}{\partial H}(H, t, \theta), \\
\frac{d \rho}{d \theta}=-\frac{\partial I}{\partial t}=-\epsilon^{2}\left[\pi P(x(\theta, H)) f^{\prime}(t)+\pi \frac{\partial R_{1}}{\partial t}(H, t, \theta)+\frac{\partial I_{-}}{\partial t}(H, t, \theta)\right],
\end{array}\right.
$$

which is also a Hamiltonian system with the new Hamiltonian function

$$
\Gamma(t, \rho, \theta ; \epsilon)=\rho+\pi \epsilon^{-2} P\left(x\left(\theta, \epsilon^{-2} \rho\right)\right) f(t)+\pi \epsilon^{-2} R_{1}\left(\epsilon^{-2} \rho, \theta, t\right)+\epsilon^{-2} I_{-}\left(\epsilon^{-2} \rho, \theta, t\right) .
$$


Obviously, if $\epsilon \ll 1$, the solution $\left(t\left(\theta, t_{0}, \rho_{0}\right), \rho\left(\theta, t_{0}, \rho_{0}\right)\right)$ of $(2.15)$ with the initial data $\left(t_{0}, \rho_{0}\right) \in R \times[1,2]$ is defined in the interval $\theta \in[0,2 \pi]$ and $\rho\left(\theta, t_{0}, \rho_{0}\right) \in\left[\frac{1}{2}, 3\right]$. So, the Poincaré map of (2.15) is well defined in the domain $R \times[1,2]$.

Lemma 6 ([8] Lemma 5.1) The Poincaré map of (2.15) has the intersection property.

The proof is similar to the corresponding one in [8].

For convenience, we introduce the notation $O_{k}(1)$ and $o_{k}(1)$. We say a function $f(t, \rho$, $\theta, \epsilon) \in O_{k}(1)$ if $f$ is smooth in $(t, \rho)$ and for $k_{1}+k_{2} \leq k$,

$$
\left|\frac{\partial^{k_{1}+k_{2}}}{\partial t^{k_{1}} \partial \rho^{k_{2}}} f(t, \rho, \theta, \epsilon)\right| \leq C,
$$

for some constant $C>0$ which is independent of the arguments $t, \rho, \theta, \epsilon$.

Similarly, we say $f(t, \rho, \theta, \epsilon) \in o_{k}(1)$ if $f$ is smooth in $(t, \rho)$ and for $k_{1}+k_{2} \leq k$,

$$
\lim _{\epsilon \rightarrow 0}\left|\frac{\partial^{k_{1}+k_{2}}}{\partial t^{k_{1}} \partial \rho^{k_{2}}} f(t, \rho, \theta, \epsilon)\right|=0,
$$

uniformly in $(t, \rho, \theta)$.

\subsection{Poincaré map and twist theorems}

We will use Ortega's small twist theorem to prove that the Poincaré map $P$ has an invariant closed curve if $\epsilon$ is sufficiently small. Let us first recall the theorem in [9].

Lemma 7 (Ortega's theorem) Let $A=\mathbb{S}^{1} \times[a, b]$ be a finite cylinder with universal cover $\mathbb{A}=\mathbb{R} \times[a, b]$. The coordinate in $\mathbb{A}$ is denoted by $(\tau, v)$. Consider the map

$$
\bar{f}: A \rightarrow \mathbb{S} \times \mathbb{R}
$$

We assume that the map has the intersection property. Suppose that $f: A \rightarrow \mathbb{R} \times \mathbb{R}$, $\left(\tau_{0}, v_{0}\right) \rightarrow\left(\tau_{1}, v_{1}\right)$ is a lift of $\bar{f}$ and it has the form

$$
\left\{\begin{array}{l}
\tau_{1}=\tau_{0}+2 N \pi+\delta l_{1}\left(\tau_{0}, v_{0}\right)+\delta \tilde{g}_{1}\left(\tau_{0}, v_{0}\right), \\
v_{1}=v_{0}+\delta l_{2}\left(\tau_{0}, v_{0}\right)+\delta \tilde{g}_{2}\left(\tau_{0}, v_{0}\right),
\end{array}\right.
$$

where $N$ is an integer, $\delta \in(0,1)$ is a parameter. The functions $l_{1}, l_{2}, \tilde{g}_{1}$ and $\tilde{g}_{2}$ satisfy

$$
\begin{aligned}
& l_{1} \in C^{6}(A), \quad l_{1}\left(\tau_{0}, v_{0}\right)>0, \quad \frac{\partial l_{1}}{\partial v_{0}}\left(\tau_{0}, v_{0}\right)>0, \quad \forall\left(\tau_{0}, v_{0}\right) \in A, \\
& l_{2}(\cdot, \cdot), \tilde{g}_{1}(\cdot, \cdot, \epsilon), \tilde{g}_{2}(\cdot, \cdot, \epsilon) \in C^{5}(A) .
\end{aligned}
$$

In addition, we assume that there is a function $I: A \rightarrow R$ satisfying

$$
I \in C^{6}(A), \quad \frac{\partial I}{\partial \nu_{0}}\left(\tau_{0}, \nu_{0}\right)>0, \quad \forall\left(\tau_{0}, \nu_{0}\right) \in A
$$

and

$$
l_{1}\left(\tau_{0}, v_{0}\right) \cdot \frac{\partial I}{\partial \tau_{o}}\left(\tau_{0}, v_{0}\right)+l_{2}\left(\tau_{0}, v_{0}\right) \cdot \frac{\partial I}{\partial \nu_{0}}\left(\tau_{0}, v_{0}\right)=0, \quad \forall\left(\tau_{0}, v_{0}\right) \in A
$$


Moreover, suppose that there are two numbers $\tilde{a}$ and $\tilde{b}$ such that $a<\tilde{a}<\tilde{b}<b$ and

$$
I_{M}(a)<I_{m}(\tilde{a}) \leq I_{M}(\tilde{a})<I_{m}(\tilde{b}) \leq I_{M}(\tilde{b})<I_{m}(b),
$$

where

$$
I_{M}(r)=\max _{\rho \in S^{1}} I\left(\rho_{o}, \tau_{o}\right), \quad I_{m}(r)=\min _{\rho \in S^{1}} I\left(\rho_{o}, \tau_{o}\right) .
$$

Then there exist $\epsilon>0$ and $\Delta>0$ such that if $\delta<\Delta$ and

$$
\left\|\tilde{g}_{1}(\cdot, \cdot, \epsilon)\right\|_{C^{5}(A)}+\left\|\tilde{g}_{2}(\cdot, \cdot, \epsilon)\right\|_{C^{5}(A)}<\epsilon,
$$

the mapping $\bar{f}$ has an invariant curve in $\Gamma_{A}$, the constant $\epsilon$ is independent of $\delta$.

We make the ansatz that the solution of (2.15) with the initial condition $(t(0), \rho(0))=$ $\left(t_{0}, \rho_{0}\right)$ is of the form

$$
t=t_{0}+\theta+\epsilon^{1-\alpha} \Sigma_{1}\left(t_{0}, \rho_{0}, \theta ; \epsilon\right), \quad \rho=\rho_{0}+\epsilon^{1-\alpha} \Sigma_{2}\left(t_{0}, \rho_{0}, \theta ; \epsilon\right) .
$$

Then the Poincaré map of (2.15) is

$$
P: \quad t_{1}=t_{0}+2 \pi+\epsilon^{1-\alpha} \Sigma_{1}\left(t_{0}, \rho_{0}, 2 \pi ; \epsilon\right), \quad \rho_{1}=\rho_{0}+\epsilon^{1-\alpha} \Sigma_{2}\left(t_{0}, \rho_{0}, 2 \pi ; \epsilon\right) .
$$

The functions $\Sigma_{1}$ and $\Sigma_{2}$ satisfy

$$
\left\{\begin{aligned}
\Sigma_{1}= & \pi \epsilon^{\alpha-1} \int_{0}^{\theta} \frac{\partial x}{\partial H}\left(\epsilon^{-2} \rho, \theta\right) p\left(x\left(\epsilon^{-2} \rho, \theta\right)\right) f(t) d \theta \\
& +\pi \epsilon^{\alpha-1} \int_{0}^{\theta}\left(\frac{\partial R_{1}}{\partial H}\left(\epsilon^{-2} \rho, t, \theta\right)+\frac{\partial I}{\partial H}\left(\epsilon^{-2} \rho, t, \theta\right)\right) d \theta, \\
\Sigma_{2}= & -\pi \epsilon^{\alpha+1} \int_{0}^{\theta} P\left(x\left(\epsilon^{-2} \rho, \theta\right)\right) f^{\prime}(t) d \theta \\
& -\epsilon^{\alpha+1} \int_{0}^{\theta}\left(\pi \frac{\partial R_{1}}{\partial t}\left(\epsilon^{-2} \rho, t, \theta\right)-\frac{\partial I}{\partial t}\left(\epsilon^{-2} \rho, t, \theta\right)\right) d \theta,
\end{aligned}\right.
$$

where $t=t_{0}+\theta+\epsilon^{1-\alpha} \Sigma_{1}, \rho=\rho_{0}+\epsilon^{1-\alpha} \Sigma_{2}$. By Lemmas 4, 6 and 7, we know that

$$
\left|\Sigma_{1}\right|+\left|\Sigma_{2}\right| \leq C \quad \text { for } \theta \in[0,2 \pi] .
$$

Hence, for $\rho_{0} \in[1,2]$, we may choose $\epsilon$ sufficiently small such that

$$
\rho_{0}+\epsilon \Sigma_{2} \geq \frac{\rho_{0}}{2} \geq \frac{1}{2} .
$$

Moreover, we can prove that

$$
\Sigma_{1}, \Sigma_{2} \in O_{6}(1)
$$

Similar to the way of estimating $R_{1}$, by a direct calculation, we have the following lemma. 
Lemma 8 The following estimates hold:

$$
\begin{aligned}
& P\left(x\left(\epsilon^{-2} \rho, \theta\right)\right)-P\left(x\left(\epsilon^{-2} \rho_{0}, \theta\right)\right) \in \epsilon^{-\alpha} O_{6}(1), \\
& \frac{\partial x}{\partial H}\left(\epsilon^{-2} \rho, \theta\right) p\left(x\left(\epsilon^{-2} \rho, \theta\right)\right)-\frac{\partial x}{\partial H}\left(\epsilon^{-2} \rho_{0}, \theta\right) p\left(x\left(\epsilon^{-2} \rho_{0}, \theta\right)\right) \in \epsilon^{2-\alpha} O_{6}(1) .
\end{aligned}
$$

Now we turn to give an asymptotic expression of the Poincaré map of (2.14), that is, we study the behavior of the functions $\Sigma_{1}$ and $\Sigma_{2}$ at $\theta=\pi$ as $\epsilon \rightarrow 0$. In order to estimate $\Sigma_{1}$ and $\Sigma_{2}$, we need to introduce the following definition and lemma. Let

$$
\Theta_{+}(I)=\operatorname{meas}\left\{\theta \in[0, \pi], x\left(H_{0}, \theta\right)>0\right\}, \quad \Theta_{-}(I)=T_{0}-\Theta_{+}(I),
$$

where $H_{0}=\epsilon^{-2} \rho_{0}$.

\section{Lemma 9}

$$
\Theta_{+}(I)=\pi+\epsilon O_{6}(1), \quad \Theta_{-}(I)=\epsilon O_{6}(1) .
$$

Proof This lemma was proved in [7], so we omit the details.

For estimate $\Sigma_{1}$ and $\Sigma_{2}$, we need the estimates of $x$ and $x_{H}$.

We recall that when $x<0$, we have

$$
\left|x\left(H_{0}, \theta\right)\right|=O_{6}(1), \quad\left|x_{H}\left(H_{0}, \theta\right)\right|=\epsilon^{2} O_{5}(1) .
$$

When $x>0$, by the definition of $\theta$, we have

$$
\arcsin \frac{x\left(H_{0}, \theta\right)}{\sqrt{2 h}}=\frac{T_{0}(h)}{\pi} \theta-\frac{T_{-}(h)}{2}=\theta+\epsilon^{2} O_{5}(1),
$$

which yields that

$$
\begin{aligned}
& x\left(H_{0}, \theta\right)=\sqrt{\frac{2 H_{0}}{\pi}} \sin \theta+O_{5}(1), \\
& x_{H}\left(H_{0}, \theta\right)=\sqrt{\frac{1}{2 H_{0} \pi}} \sin \theta+\epsilon^{2} O_{5}(1) .
\end{aligned}
$$

Now we can give the estimates of $\Sigma_{1}$ and $\Sigma_{2}$.

Lemma 10 The following estimates hold true:

$$
\begin{aligned}
& \Sigma_{1}\left(t_{0}, \rho_{0}, 2 \pi ; \epsilon\right)=\left(\frac{\pi}{2 \rho_{0}}\right)^{\frac{\alpha-1}{2}} \int_{0}^{\pi}(\sin \theta)^{1+\alpha} f\left(t_{0}+\theta\right) d \theta+o_{6}(1), \\
& \Sigma_{2}\left(t_{0}, \rho_{0}, 2 \pi ; \epsilon\right)=-\pi^{\frac{1-\alpha}{2}}\left(2 \rho_{0}\right)^{\frac{\alpha+1}{2}} \int_{0}^{\pi}(\sin \theta)^{1+\alpha} f^{\prime}\left(t_{0}+\theta\right) d \theta+o_{6}(1)
\end{aligned}
$$

for $\epsilon \rightarrow 0$. 
Proof Firstly we consider $\Sigma_{1}$. By Lemmas 3, 4, 8 and (2.22), we have

$$
\begin{aligned}
\Sigma_{1}\left(t_{0}, \rho_{0}, 2 \pi ; \epsilon\right)= & \pi \epsilon^{\alpha-1} \int_{0}^{\pi} \frac{\partial x}{\partial H}\left(\epsilon^{-2} \rho, \theta\right) p\left(x\left(\epsilon^{-2} \rho, \theta\right)\right) f(t) d \theta \\
& +\epsilon^{\alpha-1} \int_{0}^{\pi} \pi \frac{\partial R_{1}}{\partial H}\left(x\left(\epsilon^{-2} \rho, \theta\right), t\right)+\frac{\partial I_{-}}{\partial H}\left(x\left(\epsilon^{-2} \rho, \theta\right), t\right) d \theta \\
= & \pi \epsilon^{\alpha-1} \int_{0}^{\pi} \frac{\partial x}{\partial H}\left(\epsilon^{-2} \rho_{0}, \theta\right) p\left(x\left(\epsilon^{-2} \rho_{0}, \theta\right)\right) f\left(t_{0}+\theta\right) d \theta+\epsilon^{\alpha} O_{6}(1) \\
= & \pi \epsilon^{\alpha-1} \int_{\Theta_{+}} \frac{\partial x}{\partial H}\left(\epsilon^{-2} \rho_{0}, \theta\right) p\left(x\left(\epsilon^{-2} \rho_{0}, \theta\right)\right) f\left(t_{0}+\theta\right) d \theta \\
& +\pi \epsilon^{\alpha-1} \int_{\Theta_{-}} \frac{\partial x}{\partial H}\left(\epsilon^{-2} \rho_{0}, \theta\right) p\left(x\left(\epsilon^{-2} \rho_{0}, \theta\right)\right) f\left(t_{0}+\theta\right) d \theta+\epsilon^{\alpha} O_{6}(1) .
\end{aligned}
$$

Since $\lim _{x \rightarrow+\infty} \frac{p(x)}{|x|^{\alpha}}=1$ and $\epsilon \rightarrow 0$ means $x \rightarrow \infty$, we have

$$
\begin{aligned}
& \pi \epsilon^{\alpha-1} \int_{\Theta_{+}} \frac{\partial x}{\partial H}\left(\epsilon^{-2} \rho_{0}, \theta\right) p\left(x\left(\epsilon^{-2} \rho_{0}\right), \theta\right) f\left(t_{0}+\theta\right) d \theta \\
& =\pi \epsilon^{\alpha-1} \int_{\Theta_{+}} \frac{\partial x}{\partial H}\left(\theta, \epsilon^{-2} \rho\right)|x|^{\alpha} f\left(t_{0}+\theta\right) d \theta+\epsilon^{\alpha} O_{6}(1) .
\end{aligned}
$$

By the measure of $\Theta_{-}$, we have

$$
\pi \epsilon^{\alpha-1} \int_{\Theta_{-}} \frac{\partial x}{\partial H}\left(\epsilon^{-2} \rho_{0}, \theta\right) p\left(x\left(\epsilon^{-2} \rho_{0}, \theta\right)\right) f\left(t_{0}+\theta\right) d \theta=\epsilon^{\alpha} O_{6}(1) .
$$

By (2.26) and (2.27), we have

$$
\begin{aligned}
\Sigma_{1}\left(t_{0}, \rho_{0}, 2 \pi ; \epsilon\right) & =\pi \epsilon^{\alpha-1} \int_{\Theta_{+}} \frac{\partial x}{\partial H}\left(\theta, \epsilon^{-2} \rho\right)|x|^{\alpha} f\left(t_{0}+\theta\right) d \theta+\epsilon^{\alpha} O_{6}(1) \\
& =\pi \epsilon^{\alpha-1} \int_{0}^{\pi} \frac{\partial x}{\partial H}\left(\theta, \epsilon^{-2} \rho\right)|x|^{\alpha} f\left(t_{0}+\theta\right) d \theta+\epsilon^{\alpha} O_{6}(1) \\
& =\left(\frac{\pi}{2 \rho_{0}}\right)^{\frac{1-\alpha}{2}} \int_{0}^{\pi}(\sin \theta)^{\alpha+1} f\left(t_{0}+\theta\right) d \theta+o_{6}(1) .
\end{aligned}
$$

Now we consider $\Sigma_{2}$. By Lemmas 3, 4, 8 and (2.22), we have

$$
\begin{aligned}
\Sigma_{2}\left(t_{0}, \rho_{0}, 2 \pi ; \epsilon\right)= & -\pi \epsilon^{\alpha+1} \int_{0}^{\pi} P\left(x\left(\theta, \epsilon^{-2} \rho\right)\right) f^{\prime}(t) d \theta \\
& -\epsilon^{\alpha+1} \int_{0}^{\pi}\left[\pi \frac{\partial R_{1}}{\partial t}\left(x\left(\theta, \epsilon^{-2} \rho\right), t\right)+\frac{\partial I_{-}}{\partial t}\left(x\left(\theta, \epsilon^{-2} \rho\right), t\right)\right] d \theta \\
= & -\pi \epsilon^{\alpha+1} \int_{0}^{\pi} P\left(x\left(\theta, \epsilon^{-2} \rho_{0}\right)\right) f^{\prime}\left(t_{0}+\theta\right) d \theta+\epsilon^{\alpha} O_{6}(1) \\
= & -\pi \epsilon^{\alpha+1} \int_{\Theta_{+}} P\left(x\left(\theta, \epsilon^{-2} \rho_{0}\right)\right) f^{\prime}\left(t_{0}+\theta\right) d \theta \\
& -\pi \epsilon^{\alpha+1} \int_{\Theta_{-}} P\left(x\left(\theta, \epsilon^{-2} \rho_{0}\right)\right) f^{\prime}\left(t_{0}+\theta\right) d \theta+\epsilon^{\alpha} O_{6}(1) .
\end{aligned}
$$


By (1.7) and $\epsilon \rightarrow 0$ means $x \rightarrow \infty$, we have

$$
\begin{aligned}
& -\pi \epsilon^{\alpha+1} \int_{\Theta_{+}} P\left(x\left(\theta, \epsilon^{-2} \rho_{0}\right)\right) f^{\prime}\left(t_{0}+\theta\right) d \theta \\
& =-\frac{\pi \epsilon^{\alpha+1}}{\alpha+1} \int_{\Theta_{+}}\left|x\left(\theta, \epsilon^{-2} \rho_{0}\right)\right|^{\alpha} x\left(\theta, \epsilon^{-2} \rho_{0}\right) f^{\prime}\left(t_{0}+\theta\right) d \theta+\epsilon^{\alpha} O_{6}(1) .
\end{aligned}
$$

By the measure of $\Theta_{-}$, we have

$$
-\pi \epsilon^{\alpha+1} \int_{\Theta_{-}} P\left(x\left(\theta, \epsilon^{-2} \rho_{0}\right)\right) f^{\prime}\left(t_{0}+\theta\right) d \theta=\epsilon^{\alpha} O_{6}(1) .
$$

By (2.28) and (2.29), we have

$$
\begin{aligned}
\Sigma_{2} & =-\frac{\pi \epsilon^{\alpha+1}}{\alpha+1} \int_{\Theta_{+}}\left|x\left(\theta, \epsilon^{-2} \rho_{0}\right)\right|^{\alpha} x\left(\theta, \epsilon^{-2} \rho_{0}\right) f^{\prime}\left(t_{0}+\theta\right) d \theta+\epsilon^{\alpha} O_{6}(1) \\
& =-\frac{\pi \epsilon^{\alpha+1}}{\alpha+1} \int_{0}^{\pi}\left|x\left(\theta, \epsilon^{-2} \rho_{0}\right)\right|^{\alpha} x\left(\theta, \epsilon^{-2} \rho_{0}\right) f^{\prime}\left(t_{0}+\theta\right) d \theta+\epsilon^{\alpha} O_{6}(1) \\
& =-\frac{1}{\alpha+1} \pi^{\frac{1-\alpha}{2}}\left(2 \rho_{0}\right)^{\frac{\alpha+1}{2}} \int_{0}^{\pi}(\sin \theta)^{1+\alpha} f^{\prime}\left(t_{0}+\theta\right) d \theta+o_{6}(1) .
\end{aligned}
$$

Thus Lemma 10 is proved.

\subsection{Proof of Theorem 1}

Let

$$
\begin{aligned}
& \Psi_{1}\left(t_{0}, \rho_{0}\right)=\left(\frac{\pi}{2 \rho_{0}}\right)^{\frac{1-\alpha}{2}} \int_{0}^{\pi}(\sin \theta)^{1+\alpha} f\left(t_{0}+\theta\right) d \theta \\
& \Psi_{2}\left(t_{0}, \rho_{0}\right)=-\frac{1}{\alpha+1} \pi^{\frac{1-\alpha}{2}}\left(2 \rho_{0}\right)^{\frac{\alpha+1}{2}} \int_{0}^{\pi}(\sin \theta)^{1+\alpha} f^{\prime}\left(t_{0}+\theta\right) d \theta .
\end{aligned}
$$

Then there are two functions $\phi_{1}$ and $\phi_{2}$ such that the Poincaré map of (2.15), given by (2.21), is of the form

$$
\begin{aligned}
& P: \quad t_{1}=t_{0}+2 \pi+\epsilon^{1-\alpha} \Psi_{1}\left(t_{0}, \rho_{0}\right)+\epsilon^{1-\alpha} \phi_{1}, \\
& \rho_{1}=\rho_{0}+\epsilon^{1-\alpha} \Psi_{2}\left(t_{0}, \rho_{0}\right)+\epsilon^{1-\alpha} \phi_{2},
\end{aligned}
$$

where $\phi_{1}, \phi_{2} \in o_{6}(1)$.

Since $\int_{0}^{\pi} p\left(t_{0}+\theta\right) \sin \theta d \theta>0, \forall t_{0} \in R$, we have

$$
\Psi_{1}>0, \quad \frac{\partial \Psi_{1}}{\partial \rho_{0}} \neq 0 .
$$

Let

$$
L=\frac{\rho_{0}^{-\frac{1+\alpha}{2}}}{\int_{0}^{\pi}(\sin \theta)^{1+\alpha} f\left(t_{0}+\theta\right) d \theta} .
$$


Then

$$
\frac{\partial L}{\partial t_{0}} \Psi_{1}\left(t_{0}, \rho_{0}\right)+\frac{\partial L}{\partial \rho_{0}} \Psi_{2}\left(t_{0}, \rho_{0}\right)=0 .
$$

The other assumptions of Ortega's theorem are easily verified. Hence, there is an invariant curve of $P$ in the annulus $\left(t_{0}, \rho_{0}\right) \in S^{1} \times[1,2]$, which implies the boundedness of our original equation (1.5). Then Theorem 1 is proved.

\subsection{Proof of Theorem 2}

We apply Aubry-Mather theory. By Theorem B in [11] and the monotone twist property of the Poincaré map $P$ guaranteed by $\frac{\partial \Psi_{1}}{\partial \rho_{0}}<0$, it is straightforward to check that Theorem 2 is correct.

\section{Competing interests}

The authors declare that they have no competing interests.

\section{Authors' contributions}

The article is a joint work of three authors who contributed equally to the final version of the paper. All authors read and approved the final manuscript.

\section{Author details}

${ }^{1}$ College of Sciences, Nanjing University of Technology, Nanjing, 210009, People's Republic of China. ${ }^{2}$ Department of Mathematics, Southeast University, Nanjing, 210096, People's Republic of China.

\section{Acknowledgements}

Thanks are given to referees whose comments and suggestions were very helpful for revising our paper.

Received: 3 November 2012 Accepted: 3 September 2013 Published: 07 Nov 2013

\section{References}

1. Littlewood, J: Unbounded solutions of $y^{\prime \prime}+g(y)=p(t)$. J. Lond. Math. Soc. 41, 133-149 (1966)

2. Morris, GR: A case of boundedness of Littlewood's problem on oscillatory differential equations. Bull. Aust. Math. Soc. 14, 71-93 (1976)

3. Levi, M: Quasiperiodic motions in superquadratic time-periodic potential. Commun. Math. Phys. 144, 43-82 (1991)

4. Dieckerhoff, R, Zehnder, E: Boundedness of solutions via the twist theorem. Ann. Sc. Norm. Super. Pisa, Cl. Sci. 14, 79-95 (1987)

5. Liu, B: Boundedness of solutions for equations with $p$-Laplacian and an asymmetric nonlinear term. J. Differ. Equ. 207, 73-92 (2004)

6. Cheng, C, Xu, J: Boundedness of solutions for a second-order differential equations. Nonlinear Anal. 7, 1993-2004 (2008)

7. Capietto, A, Dambrosio, W, Liu, B: On the boundedness of solutions to a nonlinear singular oscillator. Z. Angew. Math. Phys. 60(6), 1007-1034 (2009)

8. Liu, B: Quasi-periodic solutions of forced isochronous oscillators at resonance. J. Differ. Equ. 246, 3471-3495 (2009)

9. Ortega, R: Boundedness in a piecewise linear oscillator and a variant of the small twist theorem. Proc. Lond. Math. Soc. 79, 381-413 (1999)

10. Jiang, S, Fang, F: Lagrangian stability of a class of second-order periodic systems. Abstr. Appl. Anal. 2011, Article ID $106214(2011)$

11. Pei, ML: Aubry-Mather sets for finite-twist maps of a cylinder and semilinear Duffing equations. J. Differ. Equ. 113, 106-127 (1994)

10.1186/1029-242X-2013-476

Cite this article as: Jiang et al.: Boundedness of solutions for semilinear Duffing's equation with asymmetric nonlinear term. Journal of Inequalities and Applications 2013, 2013:476 